\title{
Non-associative phase separation in an evaporating droplet as a model for prebiotic compartmentalization
}

\section{Wei Guo}

The University of Hong Kong

\section{Andrew Kinghorn}

University of Hong Kong

\section{Yage Zhang}

The University of Hong Kong https://orcid.org/0000-0002-1580-8200

\section{Qingchuan Li}

The University of Hong Kong

\section{Aditi Poonam}

The University of Hong Kong

\section{Julian Tanner}

University of Hong Kong https://orcid.org/0000-0002-5459-1526

Ho Shum ( $\square$ ashum@hku.hk)

University of Hong Kong https://orcid.org/0000-0002-6365-8825

\section{Article}

Keywords: Liquid-liquid phase separation, sessile droplet, localization, microenvironment

Posted Date: August 20th, 2020

DOl: https://doi.org/10.21203/rs.3.rs-56597/v1

License: (9) This work is licensed under a Creative Commons Attribution 4.0 International License. Read Full License

Version of Record: A version of this preprint was published at Nature Communications on May 27th, 2021. See the published version at https://doi.org/10.1038/s41467-021-23410-7. 


\section{Non-associative phase separation in an evaporating droplet as a model for prebiotic compartmentalization}

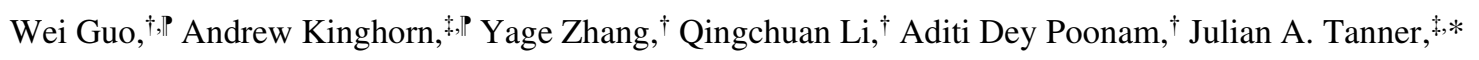

$$
\text { Ho Cheung Shum }{ }^{\dagger} *
$$

$\dagger$ Department of Mechanical Engineering, Faculty of Engineering, The University of Hong Kong,

Hong Kong (SAR), China

$\$$ School of Biomedical Sciences, LKS Faculty of Medicine, The University of Hong Kong,

Hong Kong (SAR), China

P These authors contributed equally to this work.

* To whom correspondence should be addressed:

Email: jatanner@hku.hk

ashum@hku.hk 


\begin{abstract}
Liquid-liquid phase separation (LLPS) is a mechanism by which membraneless organelles form inside cells, and has been hypothesized as a mechanism for prebiotic compartmentalization. Here we present a prebiotically plausible pathway for non-associative phase separation, which could drive biopolymer self-organization and compartmentalization to form the earliest membraneless compartments, within an evaporating all-aqueous sessile droplet. Through a quantitative understanding of the kinetic pathway of phase separation, we find that non-uniform evaporation rates across the droplet surface induce compositional gradients and surface tension differences, triggering LLPS and polymer self-organization inside the sessile droplet. With the ability to undergo LLPS, the drying droplets provide a robust mechanism for formation of prebiotic membraneless compartments. These self-organized membraneless compartments, with volumes comparable to subcellular structures, could represent a mechanism for the enrichment of information-carrying molecules and formation of RNA-containing organelles. We demonstrated compartmentalized localization and storage of nucleic acids, in vitro transcription, as well as a three-fold enhancement of ribozyme activity. This model system demonstrates a cell-like, aqueous, macromolecular crowded and thermodynamically non-equilibrium microenvironment. Such a mechanism for compartmentalization is feasible on wet organophilic silica-rich surfaces during early molecular evolution.
\end{abstract}




\section{Introduction}

Microdroplets have been demonstrated as prebiotically plausible reactors ${ }^{1-3}$. Microdroplet reactors facilitate thermodynamically unfavourable synthetic reactions in bulk solution, where one product molecule is formed from two or more reactant molecules with a high loss of entropy. ${ }^{4}$ In particular, evaporation could have played a critical role in these prebiotically relevant process, such as concentrating reagents to enhance the reaction rates inside microdroplets, ${ }^{5-7}$ and regulating conditions for biopolymer synthesis through wet-dry cycling. ${ }^{8-}$ ${ }^{10}$ Despite overcoming the inherent thermodynamic unfavorability in chemical synthesis, how prebiotic synthesized polymers assemble into cellular and subcellular-like compartments capable of autocatalysis ${ }^{11}$ and self-replication ${ }^{12,13}$ remains unresolved.

Liquid-liquid phase separation (LLPS) has recently been shown as the route for the formation of the intracellular membraneless organelles ${ }^{14-16}$. Besides their biological functions in modern cells, the existence of the membraneless organelles in cells provides a new perspective on prebiotic compartments on the early Earth, ${ }^{17}$ suggesting that these nonmembrane-bound compartments may represent "remnants" of ancient structures for spatiotemporally regulated biochemical reactions. ${ }^{18}$ This concept dates back to Alexander Oparin's arguments in the 1930s. ${ }^{19}$ LLPS is a thermodynamically transient, non-equilibrium process leading to phase segregation (non-associative, or segregative phase separation) or the formation of coacervates (associative phase separation) in an initially well-mixed macromolecular-containing systems, which can be tuned by varying polymer concentration, charge density, $\mathrm{pH}$ and temperature. With the ability to concentrate biopolymers and form primordial compartments in the dilute "primordial soup", ${ }^{20}$ LLPS has been reported to guide the transition from prebiotically synthesized polymers to highly organized structures that drive the evolutionary engine of the first living cells under prebiotic conditions. ${ }^{21}$

Currently, most proposed prebiotically plausible membraneless compartments are polyelectrolyte-rich coacervate droplets formed by associative LLPS triggered by specific interactions (such as electrostatics, cation $-\pi$ interactions, dipole-dipole contacts as well as $\pi-$ $\pi$ stacking $^{22}$ ) of oppositely charged polymers. A unique advantage of coacervate droplet formation as a prebiotic pathway of compartmentalization is its spontaneous assembly in dilute solution. Coacervate droplets could help transition from prebiotically synthesized biopolymers to fatty acid membranes encapsulated protocells. ${ }^{23-25}$ However, limitations still exist for complex coacervates as prebiotic reactors, including that structure stability and functionality of RNA could be largely inhibited due to the complex ion-pairing interactions between RNA and polycations in coacervate droplets. For example, RNA polymerization has shown to be 
inhibited in polyamines-containing coacervates ${ }^{26}$ and the rate constant of RNA cleavage is 60fold slower inside complex coacervates than that in buffers. ${ }^{27}$ In contrast, similar RNA catalytic reaction exhibits an increased rate of reaction by 70 -fold through segregative phase separation of polyethylene glycol (PEG)/dextran aqueous two-phase system (ATPS). ${ }^{28}$ It has also been reported that nucleic acid duplexes can be destabilized inside complex coacervate droplets. ${ }^{29}$ Hence, a prebiotically plausible pathway for non-associative phase separation of two neutral polymers would provide an important missing piece of the puzzles of the prebiotic compartmentalization. However, the prebiotic pathway for emergence of non-associative phase separation faces a major challenge to reach such high polymer concentrations in the dilute and homogenous "primordial soup". 30

In the free energy landscape, non-associative phase separation occurs when the mixing entropy is overcome by non-specific molecular interactions and thus polymer incompatibility appears in the solution. ${ }^{31,32}$ Hence, to trigger non-associative phase separation in the dilute solution, additional non-equilibrium settings (NES) must be introduced to enhance the nonspecific molecular interactions or reduce mixing entropy. NES has been widely applied in prebiotic chemistry to maintain thermodynamically unfavored processes such as accumulation of biomolecules. ${ }^{33-35} \mathrm{~A}$ very recent example is that, to mimic molecular evolution within porous volcanic rocks on the early Earth, heated microbubbles were used as the reactors for assembly and localization of prebiotic molecules. ${ }^{36}$ Nevertheless, how the non-associative phase separation can be achieved under prebiotically plausible NES remains largely unexplored, despite that segregative LLPS of ATPS has been hypothesized to play important roles in origins of life chemistry. ${ }^{28,30,37}$

Inspired by the widely introduced evaporation process in wet-dry cycling and prebiotically plausible microdroplet reactors, here we report droplet evaporation-assisted non-associative phase separation for prebiotic compartmentalization inside a sessile droplet of ATPS. By formulating the LLPS mechanism under the NES, we show that the non-uniform evaporation flux and initial composition of the sessile droplets determine the formation and evolution of self-organized membraneless compartments. The evolution of phase-separated membraneless compartments inside the aqueous sessile droplet looks and behaves similarly to intracellular phase separation. Most importantly, the membraneless compartments formed in our model system can serve as spatially functional reactors for the enrichment of informational molecules and the flow of genetic information, validated by the in vitro transcription and enhanced ribozyme activities. Our model can validate non-associative phase separation in moderate 
conditions with no heating, cooling or osmotic shock needed. This simple yet robust system can capture both the macromolecularly crowded interior environment and the non-equilibrium features of extant cells. Hence, the model may be important for compartmentalization in early molecular evolution.

\section{Results}

\section{Non-associative phase separation inside the evaporating sessile droplet}

A single-phase mixture of PEG and dextran, which has been extensively studied in our previous work, ${ }^{38-41}$ is prepared and used as the stock solution for non-associative phase separation. To achieve the NES required for LLPS, a droplet extracted from the stock solution is pipetted onto a glass slide and starts to evaporate. Similar scenarios with such NES, that aqueous sessile droplets dispersed on the organophilic silica-rich surfaces, could be found ubiquitously on the early Earth (Figure 1(a)), which is of essential significance for prebiotic polymerization of long oligomers. ${ }^{42-44}$ Moreover, it has recently been demonstrated that the evaporation on wavewetted rocks near the carbonate-rich lakes would increase phosphate concentration to the molar level, a critical concentration for laboratory prebiotic synthesis. ${ }^{45}$ Hence, by sharing similar prebiotically plausible conditions mentioned above, we show that non-associative phase separation for polymer self-organization and compartmentalization could also be achieved through droplet evaporation (Figure 1(a)).

Figure 1(c) and (d) show the image sequence of phase separation and polymer selforganization within the evaporating sessile droplet. Initially, the sessile droplet exhibits a homogeneous fluorescence intensity, indicating a single-phase mixture and a lack of compartments inside the droplet. Over time, the green fluorescence domains gradually appear, indicating the occurrence of phase separation and formation of the dextran-rich compartments. With different initial concentrations of polymers dissolved in the droplet, there are two distinct morphologies of self-organized patterns of polymer phase separation, as shown by the green fluorescence profile inside the droplet. For a sessile droplet consisting of $5 \mathrm{wt} \%$ PEG and 10 wt\% dextran (Regime 1, Figure 1(b1)), a lobe-shaped dextran-rich phase forms shortly after the evaporation begins (Figure 1(c1) and (c2), Video S1). For the droplet consisting of $9 \mathrm{wt} \%$ PEG and 4 wt\% dextran (Regime 2, Figure 1(b2)), there is no such lobe-shaped area; instead hundreds of dispersed dextran-rich droplets are formed (Figure 1(d1) and (d2), Video S2), mainly due to the relatively lower initial concentration of dextran in this regime. 


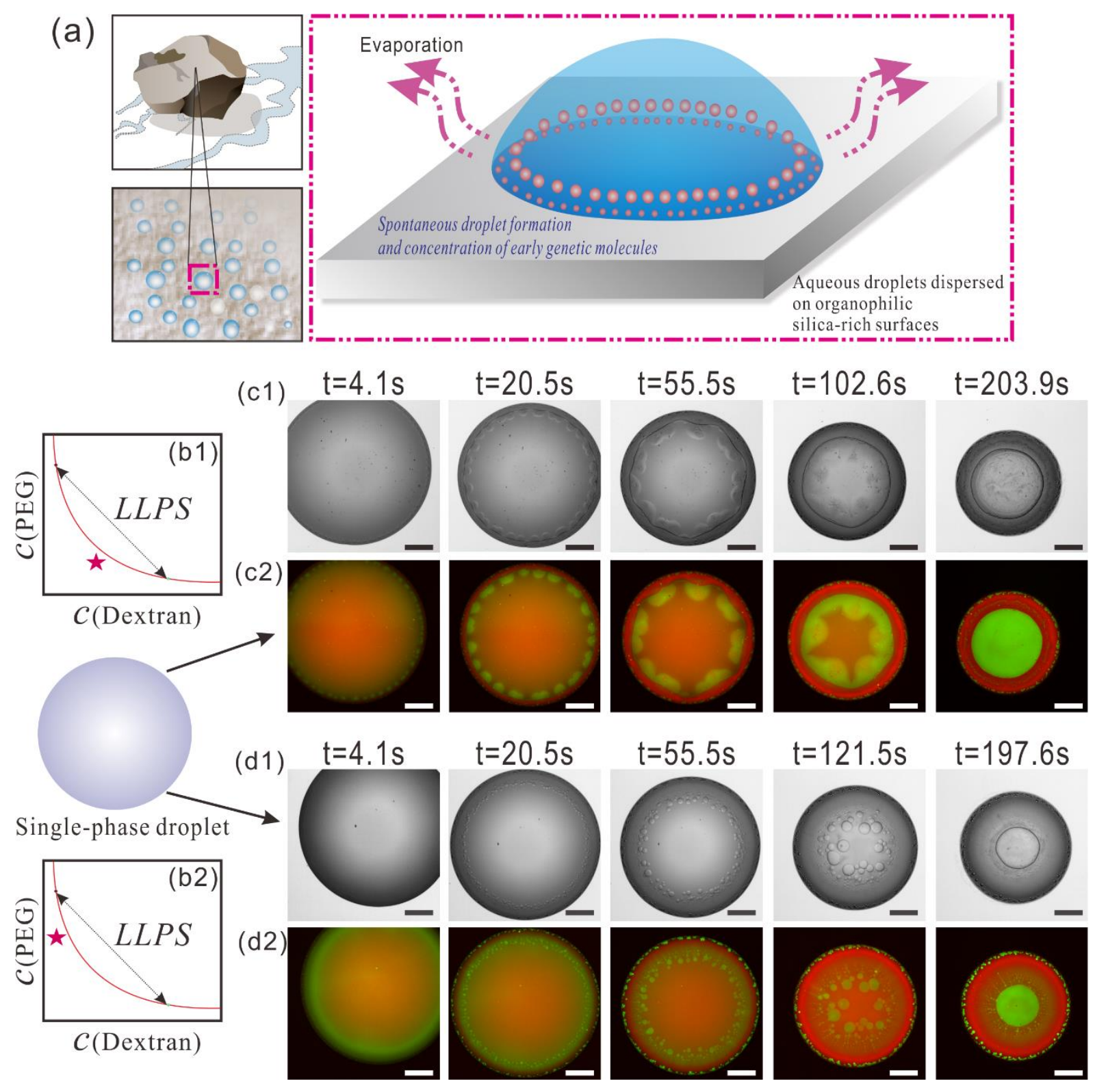

Figure 1. Evaporation-triggered segregative LLPS inside the all-aqueous sessile droplet. (a) Schematic drawing of early genetic molecule compartmentalization inside the evaporating aqueous droplets. According to timeline of the early history of life, the Pre-RNA world and RNA world may have occurred at 3.8-4.0 Gya, ${ }^{46}$ with the first RNA polymers formed in warm little ponds. ${ }^{47}$ (b) Phase diagrams of PEG and dextran mixtures; (c) Phase-separated pattern evolution of regime 1, shown in bright field image sequence (c1) and fluorescence image sequence (c2), respectively; (d) Phase-separated pattern evolution of regime 2, shown in bright field image sequence (d1) and fluorescence image sequence (d2), respectively. The scale bar is $500 \mu \mathrm{m}$.

Despite the distinct main LLPS patterns observed, the two regimes share many similar characteristics during the entire droplet evaporation process. Just at the beginning of evaporation, the sessile droplet is well mixed with a single-phase composition, as revealed by the uniform single color at the bottom. After a few seconds, small dextran-rich compartments with green fluorescence start to nucleate at the rim of the droplet. In regime 1, the dextran-rich compartments coalesce quickly after nucleation, forming lobe-shaped domains of dextran-rich 
phase (Figure 1(c2)). In regime 2, these small compartments remain as dispersed droplets (Figure 1(d2)). During further evaporation, these nucleated dextran-rich compartments, whether in the form of the lobe-shaped domains or small droplets, keep coarsening and growing, while they are hydrodynamically advected to the middle of the sessile droplet. Eventually, the dextran-rich phase covers the inner part of the sessile droplet until the evaporation process stops.

In our model system, both dextran-rich and PEG-rich domains nucleate quickly at the rim of the droplet due to phase separation. However, since PEG is more hydrophobic than dextran ${ }^{48}$ and can adsorb onto silica, ${ }^{49}$ the dextran-rich compartments, though with a higher density than PEG-rich phase, ${ }^{38}$ are advected to the inner part by the fluid flow and PEG-rich compartments stay outside the sessile droplet, as suggested by the SEM images of the deposits (SI Appendix, Fig. S1).

\section{Kinetic pathway of non-associative phase separation under the NES}

As can be seen from the experimental results, under the NES of droplet evaporation, polymer concentrations play an important role in determining LLPS patterns and their evolution (Figure 1(b)). We then test sessile droplets containing ATPS mixtures with different concentrations of PEG and dextran. In all the test, polymers are dissolved with a concentration below the binodal curve so that they are homogenously in a single phase before evaporation. The phase diagram of evaporation induced LLPS patterns for these mixtures is then obtained (Figure 2b). In general, for droplets with the concentration of dextran lower than $4 \mathrm{wt} \%$, evaporation triggers dispersed LLPS patterns (regime 2) when the concentration of PEG is more than two times higher than that of dextran. For droplets with dextran concentration higher than $4 \mathrm{wt} \%$, there is a critical concentration of PEG (about $7 \mathrm{wt} \%$ ) to determine LLPS regimes. We can observe dispersed LLPS patterns (regime 2) when the concentration of PEG is higher than this value. Otherwise, lobe-shaped LLPS patterns (regime 1) will appear. More importantly, as shown in the phase diagram of LLPS patterns (Figure 2b), polymer self-organization can be triggered by water evaporation with very little amount of PEG and dextran additives (about $1 \mathrm{wt} \%$ ), thus providing a robust strategy for prebiotic compartmentalization.

The phase diagram indicates that initial polymer concentration is an important factor that controls LLPS patterns (Figure 2 (a) and (b)). As a first qualitative understanding, water loss due to evaporation will induce the increase of polymer concentrations inside the droplet, which enhances non-specific molecular interactions and triggers polymer incompatibility. As a result, phase-separated domains are nucleated inside the droplets and coalesce into PEG-/dextran-rich 
phase-separated regions. The phase-separated regions start to appear at the rim of the sessile droplet due to the higher local evaporation rate, and they evolve with further water loss to form the LLPS patterns.

To obtain a quantitative analysis of the non-associative phase separation triggered by evaporation, we calculate the evaporation rate of the droplet to obtain the change of polymer concentrations, which determines kinetic pathway of phase separation. Evaporation induced phase segregation inside the evaporating sessile droplet of a binary and ternary mixture, with organic components involved, has been reported recently. ${ }^{50,51}$ For a sessile droplet of pure liquid, its evaporation process is dominated by vapor diffusion from the liquid-air interface to the surroundings. The analytical model of pure sessile droplet evaporation has been solved and shown as an accurate description of the evaporation dynamics. ${ }^{52}$ In our model system, despite the complex LLPS phenomena inside the evaporating droplet, the evaporation process is indeed still governed by the vapor diffusion from droplet surface to surroundings, ${ }^{50}$ so we adopt the evaporation model for a pure sessile droplet to describe the water loss process in our experiments. The mass change rate of an evaporating water droplet depends on the droplet radius $R$, vapor diffusion coeffcient $D$, saturated vapor concentration $n_{s}$, and ambient vapor concertation $n_{\infty}$, given by

$$
\frac{d M}{d t}=-\pi R D\left(n_{s}-n_{\infty}\right) f(\theta)
$$

where

$$
f(\theta)=\frac{\sin \theta}{1+\cos \theta}+4 \int_{0}^{\infty} \frac{1+\cosh 2 \theta \tau}{\sinh 2 \pi r} \tanh [(\pi-\theta) \tau] d \tau
$$

is the function of droplet contact angle $\theta$. To test this assumption, we measure the evaporation rate of a pure water droplet and a droplet of the aqueous solution containing $10 \mathrm{wt} \%$ PEG and $5 \mathrm{wt} \%$ dextran (SI Appendix, Fig. S2 and S3). The rate of mass change of both sessile droplets shows very good agreement and is consistent with the theoretical model, indicating that our assumption is reasonable. Hence, we can estimate the non-uniform evaporation rate and flux of the droplets in our experiments based on the evaporation model of a sessile droplet of pure water. 

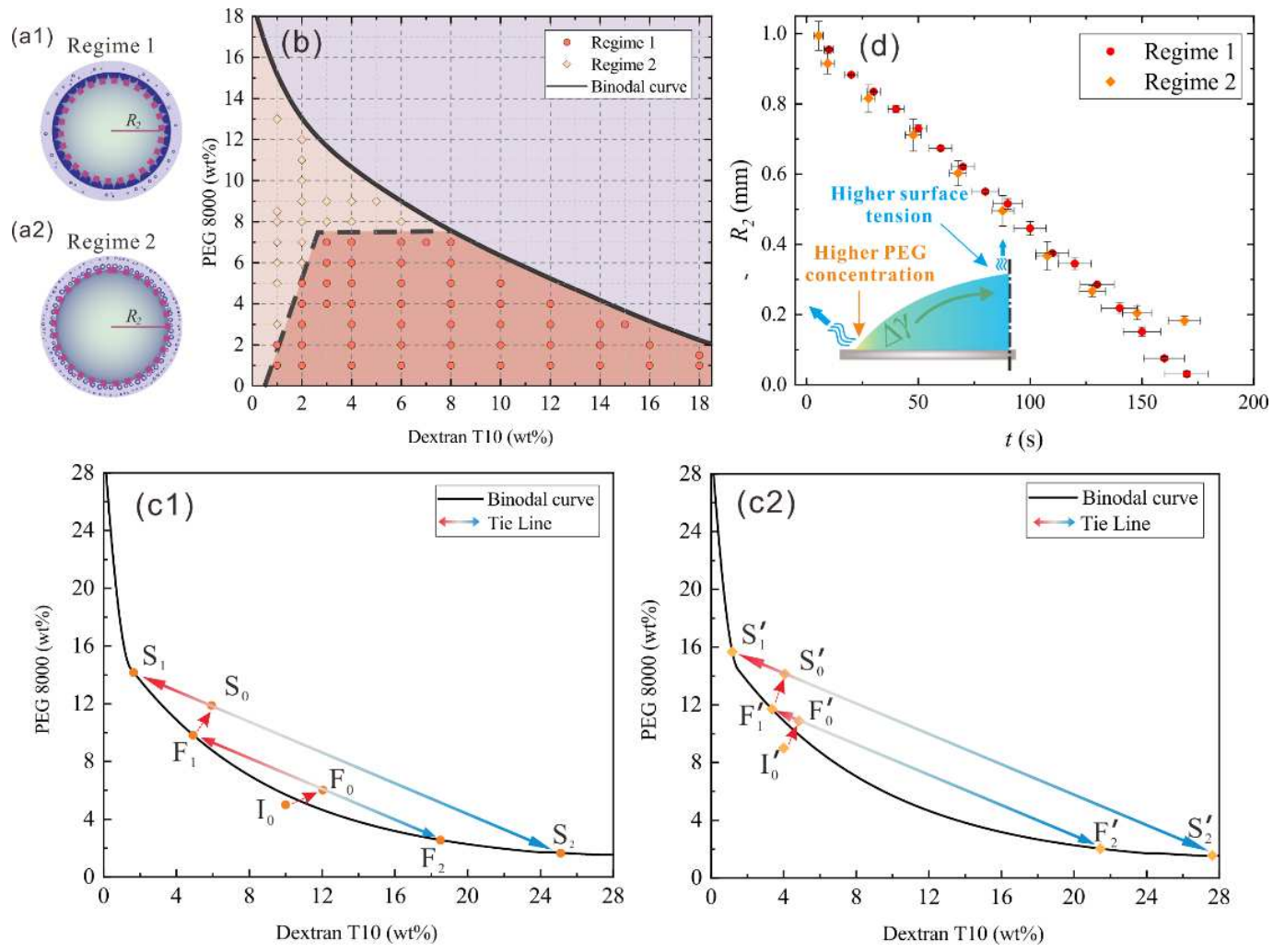

Figure 2. (a) Schematic drawings for polymer self-organized patterns in regime 1 (a1) and regime 2 (a2), with the definition of LLPS front in both regimes; (b) Phase diagram of evaporation-triggered polymer selforganization regimes inside the sessile droplet; (c) Kinetic pathway of LLPS in regime 1 (c1) and regime 2 (c2). (d) The radius of LLPS front as a function of evaporation time; Insert: Schematic drawing of non-uniform evaporation rate induced Marangoni effects.

LLPS begins at the rim of the sessile droplet because of the much higher evaporation flux here than that in the middle part of the droplet, as suggested by the evaporation flux distribution $J(r) \propto\left(R_{0}-r\right)^{-0.5}$, where $R_{0}$ is the initial droplet radius and $r$ is the horizontal distance to center of the sessile droplet. ${ }^{52}$ Due to the higher evaporation flux near the rim of the droplet (SI Appendix, Fig. S4), concentrations of PEG and dextran rapidly increase and cross over the binodal curve quickly, triggering LLPS and formation of phase-separated compartments. To simplify the problem, we select a finite small area $\left(\Delta r=0.05 R_{0}\right)$ near droplet edge as the calculation domain and estimate the increase in the polymer concentration within this domain. For both LLPS regimes demonstrated in Figure 1, phase-separated compartments are clearly visible within a few seconds. Hence, to supplement the experimental findings and generalize the understanding of LLPS, we estimate the kinetic pathway based on the polymer concentration increase in the calculation domain with a time step $(\Delta t=5 \mathrm{~s})$. The detailed calculation is given in the SI Appendix (SI Appendix, S2).

The calculated local polymer concentration increase induced by evaporation, and the corresponding kinetic pathways of the two regimes are shown in Figure 2(c1) and (c2), 
respectively. Initially, the single-phase mixtures are homogeneous without any phase-separated compartments $\left(I_{0}\right.$ and $\left.I_{0}^{\prime}\right)$. After 5 seconds of evaporation, the polymer concentrations increase and surpass the binodal curve, triggering LLPS inside the calculation domains $\left(F_{0}\right.$ and $\left.F_{0}^{\prime}\right)$. The composition and mass fraction of separated phases, including PEG-rich $\left(F_{1}\right.$ and $\left.F_{1}^{\prime}\right)$ and dextran-rich $\left(F_{2}\right.$ and $\left.F_{2}^{\prime}\right)$, are determined by the tie line in the phase diagram. For LLPS pattern evolutions in regime 1, the ratio of mass fraction of dextran-rich phase to that of the PEG-rich phase equals to the length ratio $\overline{F_{0} F_{1}} / \overline{F_{0} F_{2}}(\approx 1.1)$, suggesting that the dextran-rich phase and the PEG-rich phase formed by the first LLPS in the calculation domain have similar mass fractions. This allows the phase-separated dextran-rich compartments to coalesce quickly and form the continuous lobe-shaped structures. For LLPS pattern evolutions in regime 2, the ratio of mass fraction of dextran-rich phase to that of PEG-rich phase equals to the length ratio $\overline{F_{0}^{\prime} F_{1}^{\prime}} \overline{F_{0}^{\prime} F_{2}^{\prime}}(\approx 0.09)$, which means that the dextran-rich phase has a mass fraction over 10 times less than that of the PEG-rich phase. The low mass fraction of the dextran-rich phase inhibits the formation of the continuous lobe-shaped structures, but leads to the formation of dispersed droplets instead. Further evaporation of the water solvent in the calculation domain enhances the LLPS in the same manner $\left(S_{i}\right.$ and $S_{i}^{\prime}, i=0,1,2$.). From the kinetic pathway of phase separation, we conclude that, besides the initial composition of the mixture, the compositional change due to water loss also affects the dynamics of LLPS in the sessile droplet.

\section{Marangoni stresses induced by the non-uniform evaporation flux.}

From pattern evolutions of LLPS in both regimes, we observe that the phase-separated dextranrich compartments always move towards the center of the sessile droplet, rather than being advected to droplet edge by outwards capillary flows, as in systems exhibiting the so-called "coffee-ring effects". 53 To quantitatively understand the dynamics of phase-separated compartments, we measure the displacement of the phase separation front (PSF) inside the evaporating droplet, which is defined as the front of the phase-separated regions inside the sessile droplets (Figure 2(a1) and (a2)). Figure 2(d) shows the radius of PSF, $R_{2}$, as a function of evaporation time. For both LLPS regimes, $R_{2}$ linearly decreases with the evaporation time, which means that the displacement of PSF, $L=R-R_{2}$, has a scaling of $L \sim t$. This is different from the typical diffusion-dominated process, where the movement of different phases is driven by the concentration gradient and $L \sim t^{1 / 2}$ is expected. ${ }^{54,55}$

We attribute this reverse "coffee-ring effects" to the Marangoni flow caused by the surface tension gradient along the surface of the sessile droplet, as shown in the insert of Figure 2(d). Our assumption is based on three facts: (i) during the evaporation process, the surface tension 
force is dominant when compared to gravitational forces, as the Bond number $B o=$ $\rho g R^{2} / \gamma \approx 0.1$, where $g=9.8 \mathrm{~m} / \mathrm{s}^{2}$ is gravity and $\gamma=64 \mathrm{mN} / \mathrm{m}$ is the surface tension of the sessile droplet; (ii) the velocity of PSF is almost constant and calculated to be about 6 $\mu \mathrm{m} / \mathrm{s}$, in the same scale as that of Marangoni-driven fronts of thin spreading films; ${ }^{56}$ (iii) as a water-soluble polymer, PEG is a surface-active agent capable of decreasing surface tension of the solution (SI Appendix, Fig. S5), while an enlarged compositional gradient inside the droplet appears immediately upon LLPS, just like the much more compositional differences between $F_{2}$ (or $F_{1}$ ) and $I_{0}$ than that between $F_{0}$ and $I_{0}$.

To estimate the Marangoni effects, we use the lubrication approximation ${ }^{57}$ to simplify the model system. By balancing the capillary pressure and Marangoni stress (SI Appendix, S3), an estimated surface tension difference $\Delta \gamma \approx 2 \mathrm{mN} / \mathrm{m}$ is sufficient to induce such an inward Marangoni flow that is comparable to the outward capillary flow. Considering that in our model system, $\Delta \gamma \approx 3.5 \mathrm{mN} / \mathrm{m}$ can be easily achieved due to the highly non-uniform evaporation flux along the surface of the sessile droplet (SI Appendix, Fig. S5), we can conclude that in both LLPS regimes, the Marangoni flow driven by the surface tension differences plays a dominant role in the movement of phase-separated compartments inside the sessile droplet.

The evaporation of the sessile droplet allows us to further study the formation of the phaseseparated compartments from the microscopic perspective. The coarsening process of these compartments is likely due to transport by the hydrodynamic flow (SI Appendix, Fig. S6A). As shown by the SEM and confocal imaging (SI Appendix, Fig. S6(C) and (D)), the diameter of the small nucleated droplets ranges from hundreds of nanometers to several microns, corresponding to a volume on the order of attoliters. The size of these compartments formed through LLPS is comparable to that of phase-separated organelles in the living cells. It is difficult to make such small sized ATPS droplets by traditional droplet microfluidics techniques. ${ }^{58}$ This provides great potential for various droplet-based bioinspired techniques in our model system. ${ }^{59-62}$

Summarizing, our results present a robust NES for non-associative phase separation inside a sessile droplet. Due to the highly non-uniform evaporation flux, phase separation is triggered at the rim of the sessile droplet, exhibiting a self-organized pattern decided by both original composition and the concentration change due to the water loss, as demonstrated by the kinetic pathway of phase separation. Meanwhile, the emergence of phase separation can enlarge the compositional gradient inside the droplet, triggering Marangoni flow that convects the phaseseparated compartments to the centre of the droplet. 


\section{Encapsulation of informational molecules through membraneless compartmentalization}

Here we point out some common features of polymer self-organization and compartmentalization in our model system with that of the intracellular LLPS: ${ }^{15}$ (i) They all undergo a transition from a homogeneous state to a phase-separated state, and that only happens above a threshold concentration of the components; (ii) they are both surrounded by the aqueous macromolecular-crowding environment and governed by non-equilibrium thermodynamics and (iii) the immiscible compartments formed by LLPS both exhibit properties, such as round morphology, fusion by contact and wetting properties, that characterize typical liquid structures. These similarities make it possible to bridge prebiotic LLPS to protocells with subcellular membraneless compartments by using our model system.

The RNA world is a leading hypothesis for the origins of life. ${ }^{63}$ As RNA is a polymer capable of both information storage and a functional catalyst, it is hypothesized to be intrinsic to the origins of life. RNA can undergo associative LLPS via complex coacervation in the presence of polycations, as it has a polyanionic backbone. Likewise, RNA building blocks NTPs can undergo LLPS along with polyphosphate, which is hypothesized to be a primordial energy molecule. ${ }^{64}$ Previous studies have shown that associative LLPS is capable of concentrating such biomolecules, however the structural stability and functionality of highly-charged biopolymers like DNA and RNA have been reported to be inhibited due to their complex coacervation with polycations. $^{26,29}$ Thus a non-associative phase separation model with plausible prebiotic conditions is required, where biopolymers can be concentrated without the loss of stability and functionality.

To explore the role of non-associative phase separation in biopolymer self-organization and compartmentalization under a prebiotic environment, we firstly introduce DNA and RNA separately into our model system. To monitor DNA localisation, a Cy-5 labelled (red fluorescence) ssDNA sequence was used in combination with FITC-dextran (green fluorescence) to label the dextran-rich phase. The DNA localized to the dextran-rich phase by LLPS (Figure 3 and Video S3). This indicates that, with the functions of polymer selforganization and compartmentalization, our model system can provide a prebiotic strategy for enrichment and encapsulation of biopolymers. Similarly, RNA localised in the dextran-rich phase when introducing the fluorescent Broccoli RNA aptamer/DFHBI-T1 pair into the model system (SI Appendix, Fig. S7 and Video S4). This is consistent with previous findings that hydrophilic components like DNA and RNA partition into dextran-rich phase. ${ }^{28,37}$ For both of the Cy-5 labelled DNA and the Broccoli RNA aptamer/DFHBI-1T, the ratio of the fluorescence intensity of dextran-rich compartments $\left(I_{d}\right)$ to that of continous phase $\left(I_{c}\right), K=I_{d} / I_{c}$, has a 
more than two-order-of-magnitude increase after $\sim 4$ minutes of evaporation, indicating effective enrichment through compartmentalization. The compartmentalization of DNA and RNA provides a strategy to inhibit their thermal diffusion, thereby supporting chemical reactions in the dilute primordial soup. This spontaneous compartmentalization and concentration show a decrease in the entropic state of DNA and RNA.

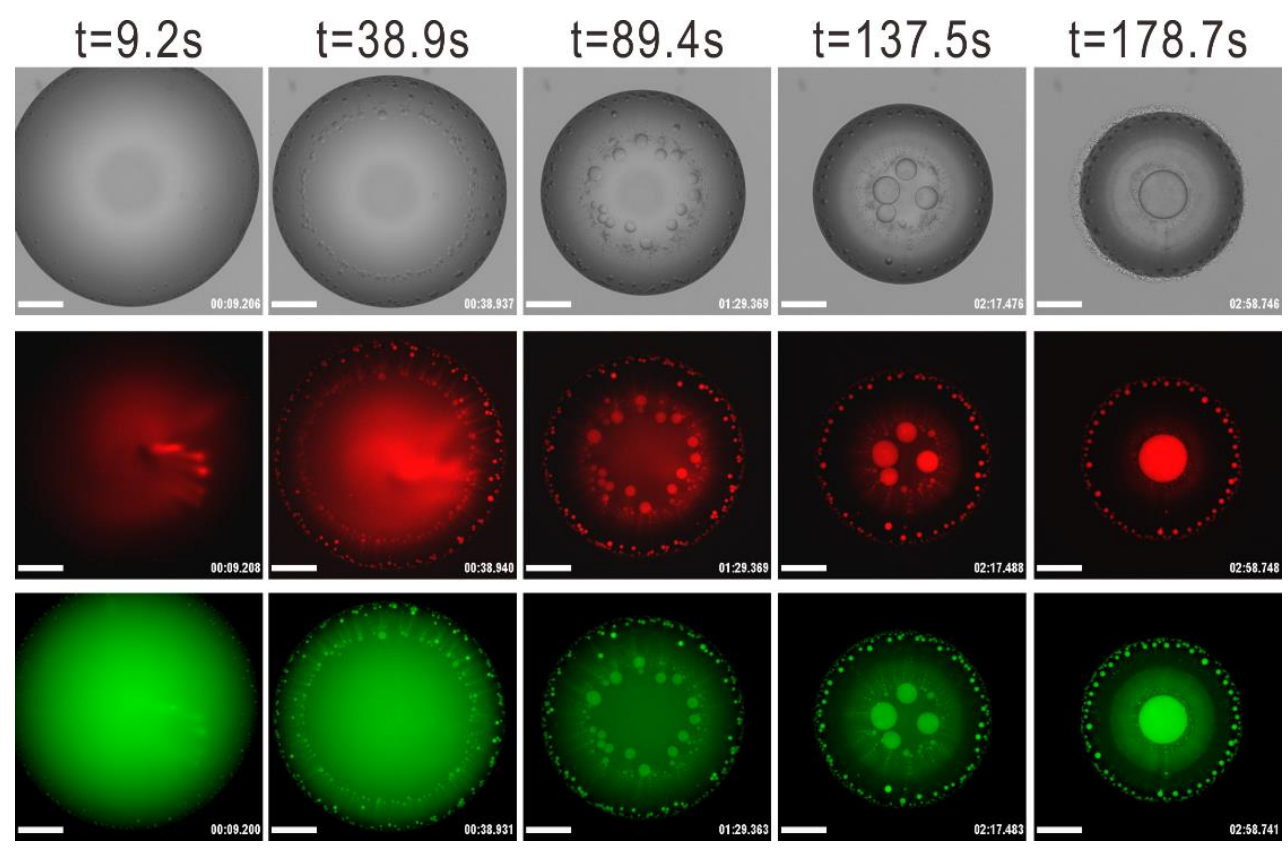

Figure 3. DNA localizes into dextran-rich compartments inside the sessile droplet. DNA is labelled with $\mathrm{Cy}-5$ (red) and dextran-rich compartments are labelled with FITC-dextran (green). The scale bar is $500 \mu \mathrm{m}$.

These DNA/RNA-containing dextran-rich liquid droplets can range from hundreds of nanometers to several microns. Similar to intracellular membrane-less compartments composed of RNA and proteins, the DNA/RNA-containing dextran-rich liquid droplets have interfacial tension-directed movement and coarsening behaviours. This implies that polymer self-organization and compartmentalization could have played a significant role in driving the evolutionary engine of the first live cells under prebiotic conditions.

To demonstrate the advantages of maintaining functionality of nucleic acids through nonassociative phase separation, we perform in vitro transcription (IVT) inside our model system (Figure 4). The sequence of confocal images shows high fluorescence in the dextran-rich compartments due to RNA synthesis, while a lower signal was observed in the surrounding PEG-rich phase. As the DNA partitions into the dextran-rich phase and there is no clear fluorescence increase outside the compartments, we conclude that transcription exclusively occurs in the phase-separated compartments. Hence, our model system can be regarded as the 
reactor that simulates the flow of genetic information under prebiotic conditions, which could have played a role in the evolution of informational polymers on the early Earth.
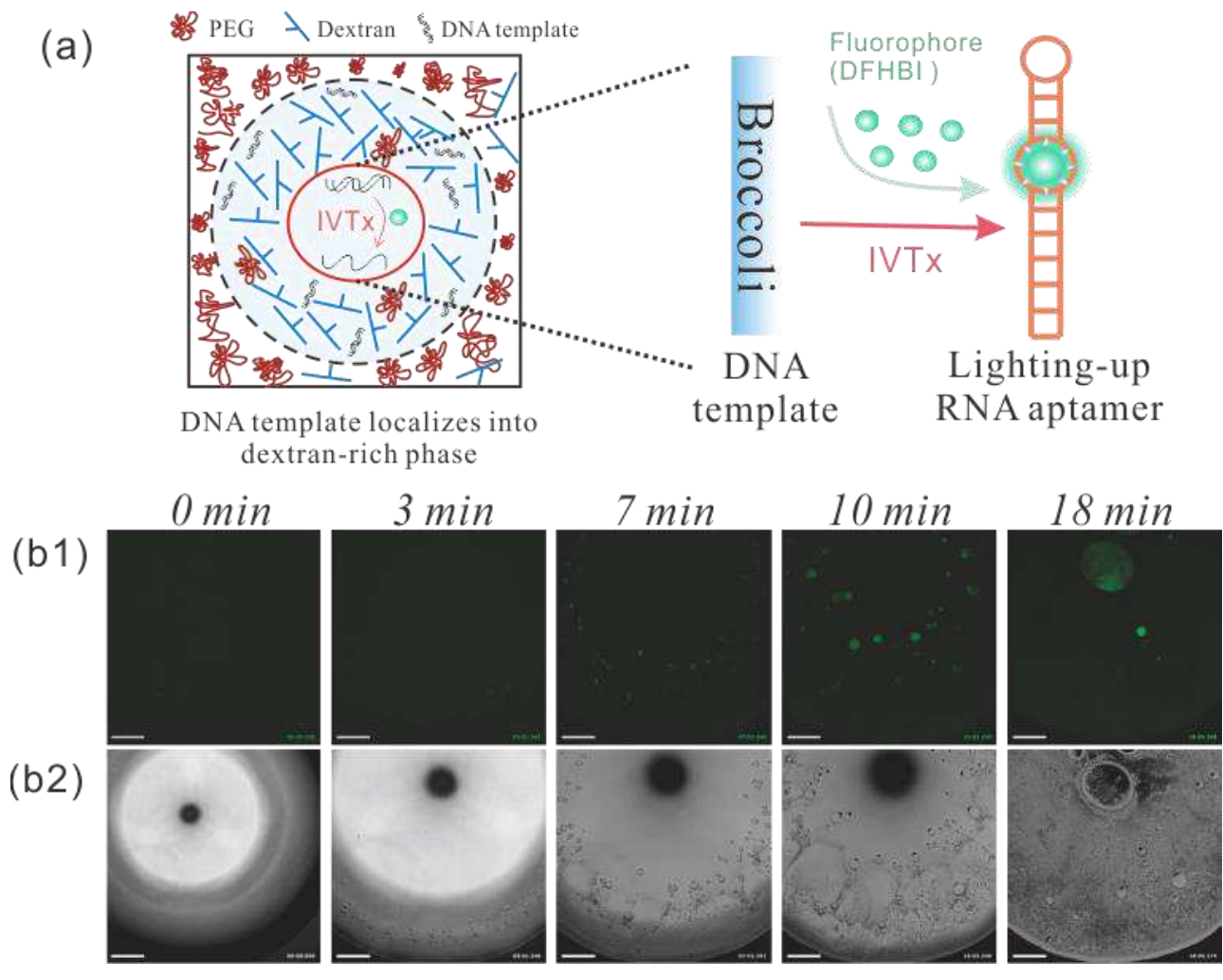

Figure 4. (a) The flow of genetic information in phase-separated compartments via in vitro transcription (IVT). (b) Optical image sequence shows fluorescence RNA aptamer transcription from Broccoli DNA template inside the phase-separated compartments. The DNA templates are coded for Broccoli aptamer that can bind 5-difluoro4-hydroxybenzylidene imidazolinone (DFHBI) to form a fluorescent complex of Broccoli-DFHBI. The scale bar is $500 \mu \mathrm{m}$.

\section{Enhanced ribozyme cleavage by compartmentalization}

According to the RNA world hypothesis, self-replicating RNA molecules underwent evolution leading to the emergence of life. ${ }^{64,65}$ Various functions of catalytic RNA under different prebiotic conditions have been demonstrated. In particular, it has been found that water evaporation would have led to a viscous environment under model prebiotic conditions and enhancing RNA replication and catalytic activities. ${ }^{66,67}$ We suggest that our model system could provide a prebiotic strategy for the concentration of catalytic RNAs and promoting their functionality. To prove this argument, we performed an RNA substrate cleavage reaction using a hammerhead ribozyme ${ }^{68}$ in the evaporating ATPS droplet. The substrate (HHS) is labelled terminally with a FAM fluorophore and a TAMRA FRET pair quencher. Under the catalysis of the hammerhead ribozyme (HHR), the substrate is cleaved into two pieces and emits fluorescence (Figure 5(a) and SI Appendix, Fig. S8). This fluorescence activation substrate 
system was used to visualize the ribozyme cleavage reaction in real time. Figure 5(b) shows the image sequence of an evaporating sessile droplet with a volume of $5 \mu \mathrm{L}$ after HHS and HHE are introduced. We can observe that catalysis occurring in fluorescent dextran-rich compartments is moving towards the center of the sessile droplet during evaporation, showing increased compartmentalization. To further prove the RNA catalysis occurs during the evaporation process, two control groups were used, "no ribozyme" and "no ATPS". For the "no ribozyme" control group where there is only HHS added without any HHR (SI Appendix, Fig. S9), we can also observe fluorescent domains in the evaporating droplet (Figure 5(c)) albeit with a lower relative fluorescence level. The pattern formed in this control group differs from the experimental group in that most of the fluorescent dextran-rich regions stay at the rim of the droplet (Figure 5(c)). For the "no ATPS" control group, both the fluorophore-labelled substrate and enzyme strand were introduced into the solution without the reagents required for ATPS (SI Appendix, Fig. S10). The results of "no ATPS" control group present much lower ribozyme cleavage activity (SI Appendix, Fig. S11), indicating the unique contributions of compartmentalization via non-associative phase separation in prebiotic chemistry.

To quantify the fluorescence levels of sessile droplets over time, we take the flattened y-axis intensity signal within the dashed red rectangle (Figure 5(b) and (c)). This provides the spatial evolution over time of the fluorescent areas in the evaporating droplet, as shown in Figure 5(d). We can see that for the experimental group, the intensity is increasing over time, with the peak of the signal moving towards the center. This is in contrast to the no ribozyme control group in which the intensity is much lower and the fluorescence signal localizes at the edge of the sessile droplets. Additionally, the no ATPS control group shows no compartmentalization and lower fluorescence (Figure 5(d)). These distinct differences not only suggest that the ribozyme cleavage reaction proceeds successfully in our model system, but that the reaction rate is increased by over 3 times (SI Appendix, Fig. S11). Taken together with the huge advantages in scalability of our model system, we conclude that this compartmentalization through nonassociative LLPS would have significant impacts in accelerations of RNA self-replications under prebiotic conditions.

To illustrate the universal plausibility of our model system to support RNA catalysis reactions, we tested another RNA ribozyme, X-motif, ${ }^{65}$ inside the evaporating sessile droplet (SI Appendix, Fig. S12). Similar cleavage reaction is achieved by evaporation induced nonassociative phase separation and compartmentalization, identifying the advantages of our 
model system in maintaining the stability and enhancing the functionality of catalytic biopolymers.

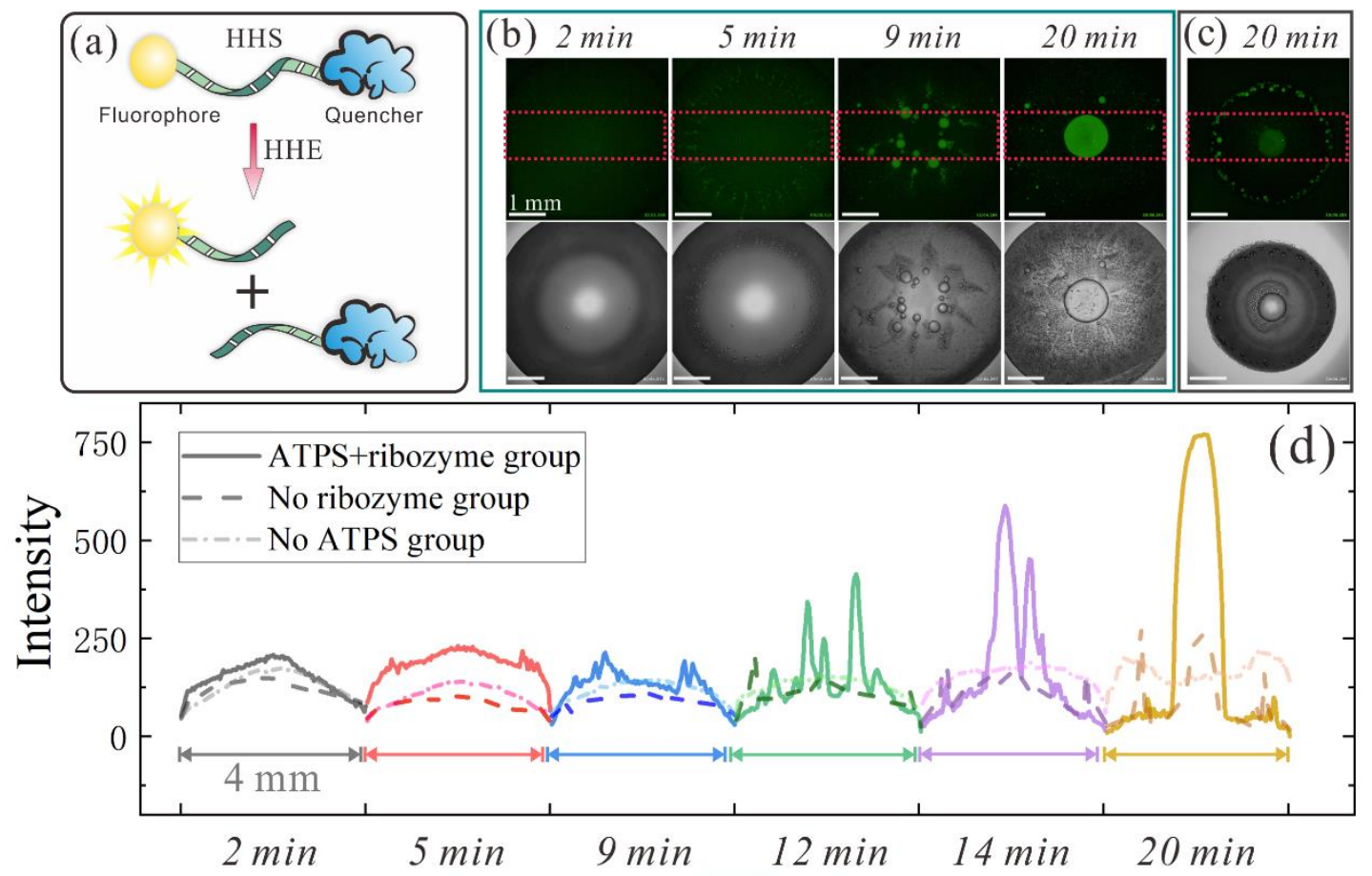

Figure 5. (a) Schematic drawing of the RNA cleavage reaction. The fluorophore labelled hammerhead substrate (HHS) is cut into two smaller pieces by the hammerhead ribozyme (HHE) and emits fluorescence. (b) Image sequence shows RNA cleavage process inside the phase-separated compartments. (c) Control group of the RNA cleavage reaction without HHE. There is only fluorophore labelled substrate added into the droplets. (d) Fluorescence intensity evolution of experimental group (solid line), no ribozyme control group (dashed line) as well as no ATPS control group (dash-dotted line). The intensity value is obtained by gel analysis of a specified rectangular area (as shown in (b) and (c)) in the fluorescence images. The scale bar is $1 \mathrm{~mm}$.

\section{Discussion}

In summary, we have presented a prebiotically plausible strategy for segregative LLPS within an evaporating sessile droplet, triggered by the NES of droplet evaporation. We quantitatively characterized the pathways of LLPS that determine the phase separation patterns. The surface tension differences induced by non-uniform evaporation flux drive the movement of LLPS front and the dynamics of nucleated compartments. Biopolymers selectively localized to the dextran-rich compartments inside the sessile droplet. We demonstrated this localization using fluorescently labelled DNA. Furthermore, we monitored the flow of genetic information in phase-separated compartments via in vitro transcription and Broccoli/DFHBI fluorescent signal. The localization and activity of ribozymes in the dextran-rich compartments inside the sessile droplet were observed. Ribozyme activity in the sessile droplets was enhanced over 3fold. The enhanced activity may be due to the increased relative concentration of the substrate 
in the dextran-rich compartments. Segregative LLPS may have been an important mechanism for compartmentalization early in molecular evolution.

\section{Methods}

Chemicals and Solutions. The sessile droplet consisted of poly(ethylene glycol) (PEG, $M_{w}=$ 8000, Sigma-Aldrich $)$ and dextran $\left(M_{w}=10000\right.$, Aladin $)$ aqueous mixture. The two polymers were dissolved in Milli-Q water. To assist fluorescent imaging, $0.01 \mathrm{mg} / \mathrm{mL}$ fluorescein isothiocyanate-dextran (4 kg/mol, Sigma-Aldrich) and $1 \mathrm{mg} / \mathrm{mL}$ Rhodamine B (>95\% (HPLC), Sigma-Aldrich) were added to the mixture. The excitation wavelength was $543 \mathrm{~nm}$. The emission was collected from 570 to $660 \mathrm{~nm}$.

Experimental Setup. A $0.5 \mu \mathrm{L}$ droplet was pipetted on a transparent microscope glass surface under room conditions (Temperature $=22{ }^{\circ} \mathrm{C}$, Humidity $=55 \%-65 \%$ ). The contact angle of the droplet varied between $45^{\circ}$ and $25^{\circ}$ during the whole evaporation process, measured by brightfield imaging (Nikon) from the side view. The microscope glass slides (ISOLAB GmbH) were used as solid substrates for the sessile droplets. The glass slides were firstly wiped by ethanol wetted tissue to mechanically get rid of contaminants on the surfaces. Then the slides were sonicated in ethanol for $15 \mathrm{~min}$, followed by successively washing with isopropyl alcohol and Milli-Q water, to remove organic contaminants on the surfaces. Then we dried the slides by nitrogen flow and put them into oven $\left(65^{\circ} \mathrm{C}\right)$ for 1 hour.

Microscope Observations. The bright field images were captured by a high-speed camera (Phantom V9.1, FASTCAM SA4, Photron) coupled with an inverted microscope (Motic AE2000). The fluorescence images were captured by a Nikon Ti2-E Widefield microscopy. The captured images are processed using ImageJ (NIH) software. Droplet deposits after fully drying on the silica wafer were sputtered with gold before imaging under scanning electron microscope (Hitachi S3400N VP, 20.0 kV).

BrocT RNA Aptamer Preparation. In vitro transcription (IVT) mixture (AS3107, Lucigen) was assembled containing $1 \times$ transcription buffer, NTPs, DTT, T7 RNA polymerase mix, 50 nM BroccoliT DNA template and $200 \mu \mathrm{M}$ DFHBI-1T. The transcription mix was incubated at $37^{\circ} \mathrm{C}$ for 1 hour to allow transcription of BroccoliT RNA. The ATPS solution is made up of 9 wt $\%$ PEG and $4 \mathrm{wt} \%$ dextran. The transcription product containing BroccoliT RNA aptamers was then added to the ATPS solution with the volume ratio of 1:10. A $0.5 \mu \mathrm{L}$ droplet of the mixture was pipetted on the glass substrate for evaporation and imaging. 
In vitro Transcription. In vitro transcription (IVT) mixture (AS3107, Lucigen) was assembled containing $1 \times$ transcription buffer, NTPs, DTT, T7 RNA polymerase mix and $200 \mu \mathrm{M}$ DFHBI1T. The ATPS solution is made up of $11 \mathrm{wt} \%$ PEG and $2 \mathrm{wt} \%$ dextran. Then the transcription mixture and $50 \mathrm{nM}$ BroccoliT DNA template solution were added into the ATPS solution with the volume ratio of 1:1:10. A $5 \mu \mathrm{L}$ droplet of the mixture was pipetted on the glass substrate for evaporation and imaging.

Ribozyme Cleavage Reaction. Both hammerhead ribozyme and X-motif ribozyme are used for the cleavage reaction inside the evaporating droplet. The ATPS solution is made up of 11 wt \% PEG and $2 \mathrm{wt} \%$ dextran. In vitro transcription (IVT) mixture (AS3107, Lucigen) was assembled containing $1 \times$ transcription buffer, NTPs, DTT, T7 RNA polymerase and $50 \mathrm{nM}$ ribozyme DNA template. The transcription mix was incubated at $37{ }^{\circ} \mathrm{C}$ for 1 hour to allow transcription of ribozyme RNA. The final mixture is prepared by adding $1 \mu \mathrm{L}$ of ribozyme substrate $(10 \mu \mathrm{M})$ and $1 \mu \mathrm{L}$ of ribozyme RNA transcription product into $38 \mu \mathrm{L}$ ATPS solution. The ribozyme reaction is started by pipetting a $5 \mu \mathrm{L}$ droplet of the final mixture on the glass substrate.

\section{Conflict of interest}

The authors declare that they have no competing interests.

\section{Acknowledgments}

We thank Dr. Zhou Liu, Dr. Youchuang Chao, Dr. Yang Xiao, Ms. Shu Ma, Ms. Danyang Ji and Mr. Fuyun Tan for very helpful discussions. This research is supported by the General Research Fund (Nos. 17304017, and 17305518) and Research Impact Fund (R7072-18), the NSFC Excellent Young Scientists Fund (Hong Kong and Macau) (21922816), the Seed Funding for Strategic Interdisciplinary Research Scheme 2017/18 from the University of Hong Kong, as well as the Sichuan Science and Technology Program (2018JZ0026).

\section{Author contributions}

W.G. and H.C.S. designed the research. W.G., Y.Z. and A.K. performed experiments. W.G. and A.K., analysed the data. W.G., A.K., J.A.T. and H.C.S. had discussions on the results and analysis. W.G., A.K., J.A.T. and H.C.S. wrote the manuscript with comments from all the coauthors. 


\section{References}

1. Dobson, C.M., Ellison, G.B., Tuck, A.F. \& Vaida, V. Atmospheric aerosols as prebiotic chemical reactors. Proc. Natl. Acad. Sci. U.S.A 97, 11864-11868 (2000).

2. Fallah-Araghi, A., et al. Enhanced chemical synthesis at soft interfaces: a universal reactionadsorption mechanism in microcompartments. Phys Rev Lett 112, 028301 (2014).

3. Ruiz-Bermejo, M., Rivas, L.A., Palacin, A., Menor-Salvan, C. \& Osuna-Esteban, S. Prebiotic synthesis of protobiopolymers under alkaline ocean conditions. Orig Life Evol Biosph 41, 331345 (2011).

4. Page, M.I. \& Jencks, W.P. Entropic Contributions to Rate Accelerations in Enzymic and Intramolecular Reactions and the Chelate Effect. Proc. Natl. Acad. Sci. U.S.A 68, 1678-1683 (1971).

5. Takáts, Z. \& Cooks, R.G. Thermal formation of serine octamer ions. Chemical Communications, 444-445 (2004).

6. Nanita, S.C. \& Cooks, R.G. Serine Octamers: Cluster Formation, Reactions, and Implications for Biomolecule Homochirality. Angew. Chem 45, 554-569 (2006).

7. Xi, H.-D., et al. Active droplet sorting in microfluidics: a review. Lab on a Chip 17, 751-771 (2017).

8. Rodriguez-Garcia, M., et al. Formation of oligopeptides in high yield under simple programmable conditions. Nat Commun 6, 8385 (2015).

9. Becker, S., et al. Wet-dry cycles enable the parallel origin of canonical and non-canonical nucleosides by continuous synthesis. Nat Commun 9, 163 (2018).

10. Campbell, T.D., et al. Prebiotic condensation through wet-dry cycling regulated by deliquescence. Nat Commun 10, 4508 (2019).

11. Chen, I.A. \& Nowak, M.A. From Prelife to Life: How Chemical Kinetics Become Evolutionary Dynamics. Accounts of Chemical Research 45, 2088-2096 (2012).

12. Sievers, D. \& von Kiedrowski, G. Self-replication of complementary nucleotide-based oligomers. Nature 369, 221-224 (1994).

13. Libicher, K., Hornberger, R., Heymann, M. \& Mutschler, H. In vitro self-replication and multicistronic expression of large synthetic genomes. Nat Commun 11, 904 (2020).

14. Banani, S.F., Lee, H.O., Hyman, A.A. \& Rosen, M.K. Biomolecular condensates: organizers of cellular biochemistry. Nat Rev Mol Cell Biol 18, 285-298 (2017).

15. Feric, M., et al. Coexisting Liquid Phases Underlie Nucleolar Subcompartments. Cell 165, 16861697 (2016).

16. Schmidt, H.B. \& Görlich, D. Transport Selectivity of Nuclear Pores, Phase Separation, and Membraneless Organelles. Trends in Biochemical Sciences 41, 46-61 (2016).

17. Jia, T.Z., et al. Membraneless polyester microdroplets as primordial compartments at the origins of life. 116, 15830-15835 (2019).

18. Hyman, A.A., Weber, C.A. \& Julicher, F. Liquid-liquid phase separation in biology. Annu Rev Cell Dev Biol 30, 39-58 (2014).

19. Oparin, A. The Origin of Life. (Morgulis, 1938).

20. Monnard, P.A. \& Walde, P. Current Ideas about Prebiological Compartmentalization. Life (Basel) 5, 1239-1263 (2015).

21. Longo, L.M., et al. Primordial emergence of a nucleic acid-binding protein via phase separation and statistical ornithine-to-arginine conversion. Proceedings of the National Academy of Sciences 117, 15731 (2020).

22. Brangwynne, Clifford P., Tompa, P. \& Pappu, Rohit V. Polymer physics of intracellular phase transitions. Nature Physics 11, 899-904 (2015).

23. Lai, Y.C. \& Chen, I.A. Protocells. Curr Biol 30, R482-R485 (2020).

24. Saha, R. \& Chen, I.A. Origin of Life: Protocells Red in Tooth and Claw. Curr Bio/ 25, R1175-1177 (2015). 
25. Dora Tang, T.Y., et al. Fatty acid membrane assembly on coacervate microdroplets as a step towards a hybrid protocell model. Nat Chem 6, 527-533 (2014).

26. Poudyal, R.R., et al. Template-directed RNA polymerization and enhanced ribozyme catalysis inside membraneless compartments formed by coacervates. Nat Commun 10, 490 (2019).

27. Drobot, B., et al. Compartmentalised RNA catalysis in membrane-free coacervate protocells. Nat Commun 9, 3643 (2018).

28. Strulson, C.A., Molden, R.C., Keating, C.D. \& Bevilacqua, P.C. RNA catalysis through compartmentalization. Nature Chemistry 4, 941-946 (2012).

29. Nott, T.J., Craggs, T.D. \& Baldwin, A.J. Membraneless organelles can melt nucleic acid duplexes and act as biomolecular filters. Nat Chem 8, 569-575 (2016).

30. Poudyal, R.R., Pir Cakmak, F., Keating, C.D. \& Bevilacqua, P.C. Physical Principles and Extant Biology Reveal Roles for RNA-Containing Membraneless Compartments in Origins of Life Chemistry. Biochemistry 57, 2509-2519 (2018).

31. Berry, J., Brangwynne, C.P. \& Haataja, M. Physical principles of intracellular organization via active and passive phase transitions. Rep Prog Phys 81, 046601 (2018).

32. De Gennes, P.-G. \& Gennes, P.-G. Scaling concepts in polymer physics, (Cornell university press, 1979).

33. Baaske, P., et al. Extreme accumulation of nucleotides in simulated hydrothermal pore systems. Proc. Natl. Acad. Sci. U.S.A 104, 9346-9351 (2007).

34. Reichl, M., Herzog, M., Götz, A. \& Braun, D. Why Charged Molecules Move Across a Temperature Gradient: The Role of Electric Fields. Physical Review Letters 112, 198101 (2014).

35. Priye, A., Yu, Y., Hassan, Y.A. \& Ugaz, V.M. Synchronized chaotic targeting and acceleration of surface chemistry in prebiotic hydrothermal microenvironments. Proceedings of the National Academy of Sciences 114, 1275 (2017).

36. Morasch, M., et al. Heated gas bubbles enrich, crystallize, dry, phosphorylate and encapsulate prebiotic molecules. Nat Chem 11, 779-788 (2019).

37. Dewey, D.C., Strulson, C.A., Cacace, D.N., Bevilacqua, P.C. \& Keating, C.D. Bioreactor droplets from liposome-stabilized all-aqueous emulsions. Nat Commun 5, 4670 (2014).

38. Chao, Y., Mak, S.Y., Rahman, S., Zhu, S. \& Shum, H.C. Generation of High-Order All-Aqueous Emulsion Drops by Osmosis-Driven Phase Separation. Small 14, 1802107 (2018).

39. Lo, H.Y., et al. Diffusion-Dominated Pinch-Off of Ultralow Surface Tension Fluids. Physical Review Letters 123, 134501 (2019).

40. Song, Y., et al. Budding-like division of all-aqueous emulsion droplets modulated by networks of protein nanofibrils. Nature Communications 9, 2110 (2018).

41. Song, Y., et al. Fabrication of fibrillosomes from droplets stabilized by protein nanofibrils at all-aqueous interfaces. Nat Commun 7, 12934 (2016).

42. Smith, J.V., Arnold, F.P., Parsons, I. \& Lee, M.R. Biochemical evolution III: Polymerization on organophilic silica-rich surfaces, crystal-chemical modeling, formation of first cells, and geological clues. Proc Natl Acad Sci U S A 96, 3479-3485 (1999).

43. Hazen, R.M. \& Sverjensky, D.A. Mineral surfaces, geochemical complexities, and the origins of life. Cold Spring Harb Perspect Biol 2, a002162-a002162 (2010).

44. Ferris, J.P., Hill, A.R., Liu, R. \& Orgel, L.E. Synthesis of long prebiotic oligomers on mineral surfaces. Nature 381, 59-61 (1996).

45. Toner, J.D. \& Catling, D.C. A carbonate-rich lake solution to the phosphate problem of the origin of life. Proceedings of the National Academy of Sciences 117, 883 (2020).

46. Joyce, G.F. The antiquity of RNA-based evolution. Nature 418, 214-221 (2002).

47. Pearce, B.K.D., Pudritz, R.E., Semenov, D.A. \& Henning, T.K. Origin of the RNA world: The fate of nucleobases in warm little ponds. Proc Natl Acad Sci U S A 114, 11327-11332 (2017).

48. Mitchison, T. Colloid osmotic parameterization and measurement of subcellular crowding. Molecular Biology of the Cell 30, 173-180 (2019). 
49. Trens, P. \& Denoyel, R. Conformation of poly(ethylene glycol) polymers at the silica/water interface: a microcalorimetric study. Langmuir 9, 519-522 (1993).

50. Kim, H. \& Stone, H.A. Direct measurement of selective evaporation of binary mixture droplets by dissolving materials. Journal of Fluid Mechanics 850, 769-783 (2018).

51. Tan, H., et al. Evaporation-triggered microdroplet nucleation and the four life phases of an evaporating Ouzo drop. Proceedings of the National Academy of Sciences of the United States of America 113, 8642-8647 (2016).

52. Popov, Y.O. Evaporative deposition patterns: Spatial dimensions of the deposit. Physical Review E 71, 036313 (2005).

53. Deegan, R., et al. Capillary Flow as the Cause of Ring Stains From Dried Liquid Drops. Nature 389, 827-829 (1997).

54. Bian, X., Kim, C. \& Karniadakis, G.E. 111 years of Brownian motion. Soft Matter 12, 6331-6346 (2016).

55. Guo, W., et al. Pressure-Driven Filling of Closed-End Microchannel: Realization of CombShaped Transducers for Acoustofluidics. Physical Review Applied 10, 054045 (2018).

56. Cazabat, A., Heslot, F., Troian, S. \& Carles, P. . Fingering instability of thin spreading films driven by temperature gradients. Nature 346, 824-826 (1990).

57. Kim, H., et al. Controlled Uniform Coating from the Interplay of Marangoni Flows and SurfaceAdsorbed Macromolecules. Physical Review Letters 116, 124501 (2016).

58. Zhu, P. \& Wang, L. Passive and active droplet generation with microfluidics: a review. Lab on a Chip 17, 34-75 (2017).

59. Wang, H., Liu, Y., Chen, Z., Sun, L. \& Zhao, Y. Anisotropic structural color particles from colloidal phase separation. Science Advances 6, eaay1438 (2020).

60. Luo, G., et al. Freeform, Reconfigurable Embedded Printing of All-Aqueous 3D Architectures. 31, 1904631 (2019).

61. Shimanovich, U., et al. Silk micrococoons for protein stabilisation and molecular encapsulation. Nature Communications 8, 15902 (2017).

62. Shimanovich, U., et al. Sequential Release of Proteins from Structured Multishell Microcapsules. Biomacromolecules 18, 3052-3059 (2017).

63. Robertson, M.P. \& Joyce, G.F. The origins of the RNA world. Cold Spring Harb Perspect Biol 4, a003608 (2012).

64. Pressman, A., Blanco, C. \& Chen, I.A. The RNA World as a Model System to Study the Origin of Life. Curr Biol 25, R953-963 (2015).

65. Tang, J. \& Breaker, R.R. Structural diversity of self-cleaving ribozymes. Proceedings of the National Academy of Sciences of the United States of America 97, 5784-5789 (2000).

66. He, C., Gállego, I., Laughlin, B., Grover, M.A. \& Hud, N.V. A viscous solvent enables information transfer from gene-length nucleic acids in a model prebiotic replication cycle. Nat Chem $\mathbf{9}$, 318-324 (2017).

67. He, C., Lozoya-Colinas, A., Gallego, I., Grover, M.A. \& Hud, N.V. Solvent viscosity facilitates replication and ribozyme catalysis from an RNA duplex in a model prebiotic process. Nucleic Acids Res 47, 6569-6577 (2019).

68. Scott, W.G., Finch, J.T. \& Klug, A. The crystal structure of an all-RNA hammerhead ribozyme: a proposed mechanism for RNA catalytic cleavage. Cell 81, 991-1002 (1995). 


\section{Figures}

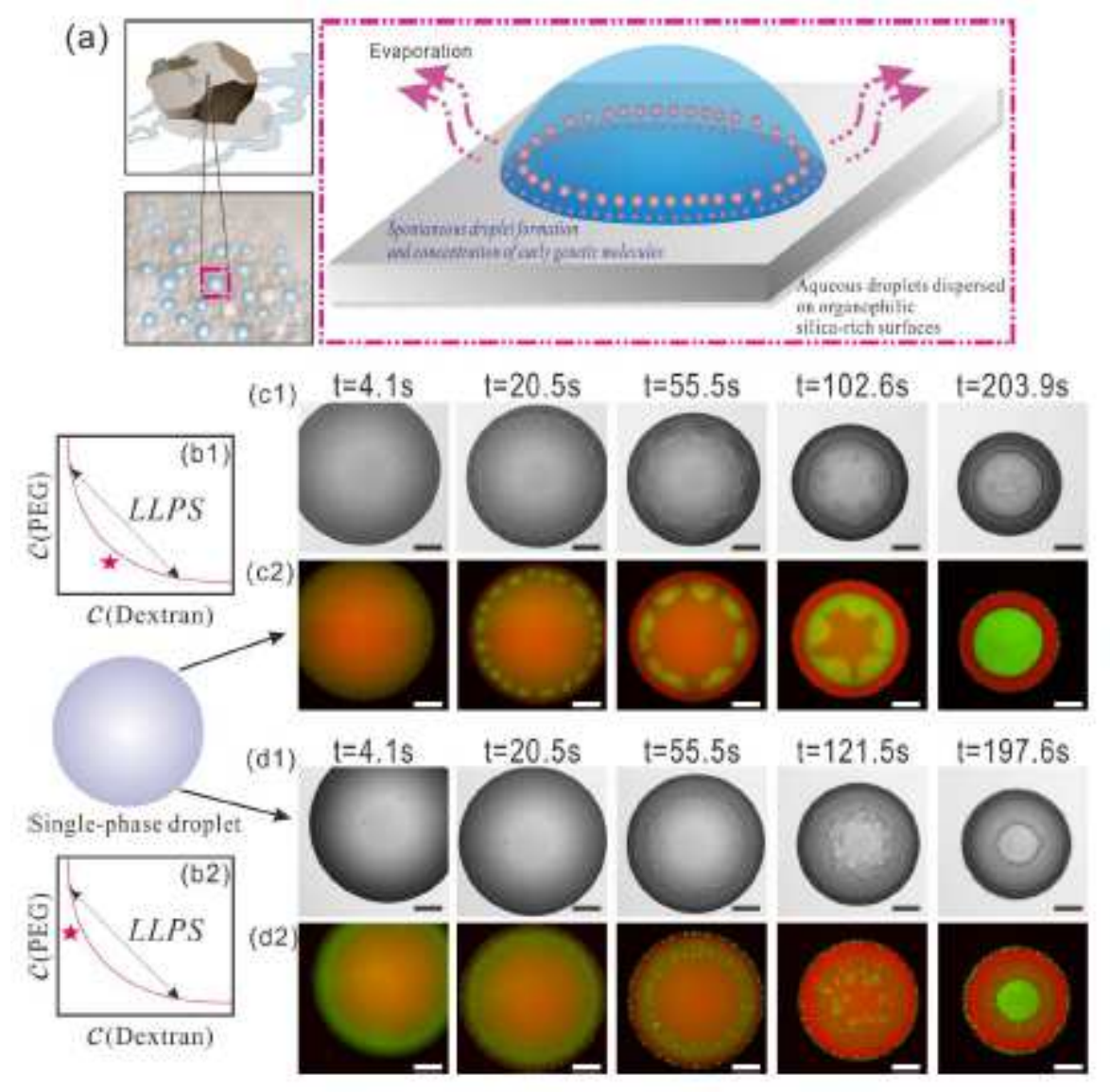

\section{Figure 1}

Evaporation-triggered segregative LLPS inside the all-aqueous sessile droplet. (a) Schematic drawing of early genetic molecule compartmentalization inside the evaporating aqueous droplets. According to timeline of the early history of life, the Pre-RNA world and RNA world may have occurred at 3.8-4.0 Gya,46 with the first RNA polymers formed in warm little ponds.47 (b) Phase diagrams of PEG and dextran mixtures; (c) Phase-separated pattern evolution of regime 1, shown in bright field image sequence (c1) and fluorescence image sequence (c2), respectively; (d) Phase-separated pattern evolution of regime 2, shown in bright field image sequence (d1) and fluorescence image sequence (d2), respectively. The scale bar is $500 \mathrm{Dm}$. 

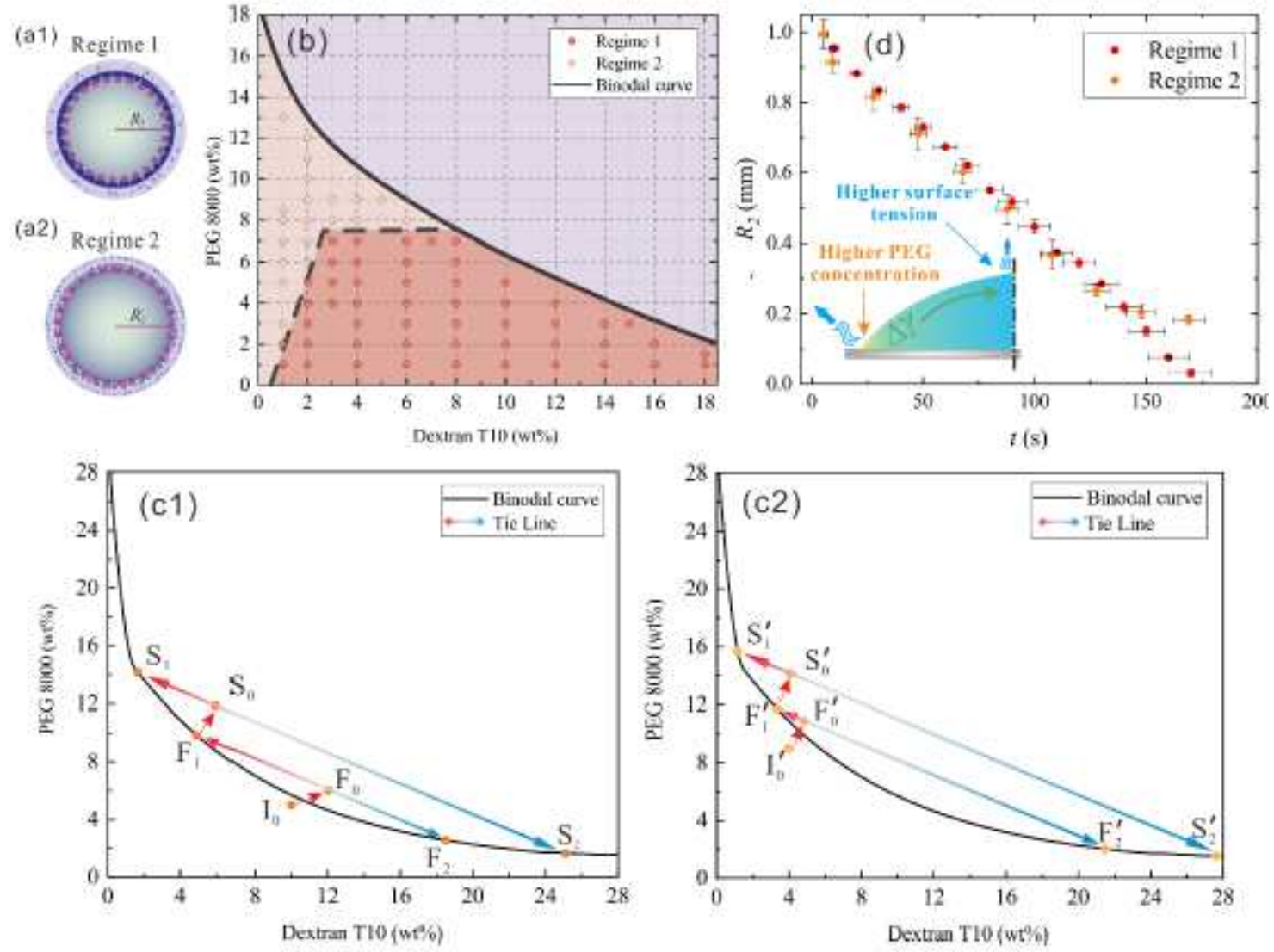

Figure 2

(a) Schematic drawings for polymer self-organized patterns in regime 1 (a1) and regime 2 (a2), with the definition of LLPS front in both regimes; (b) Phase diagram of evaporation-triggered polymer selforganization regimes inside the sessile droplet; (c) Kinetic pathway of LLPS in regime 1 (c1) and regime 2 (c2). (d) The radius of LLPS front as a function of evaporation time; Insert: Schematic drawing of nonuniform evaporation rate induced Marangoni effects. 


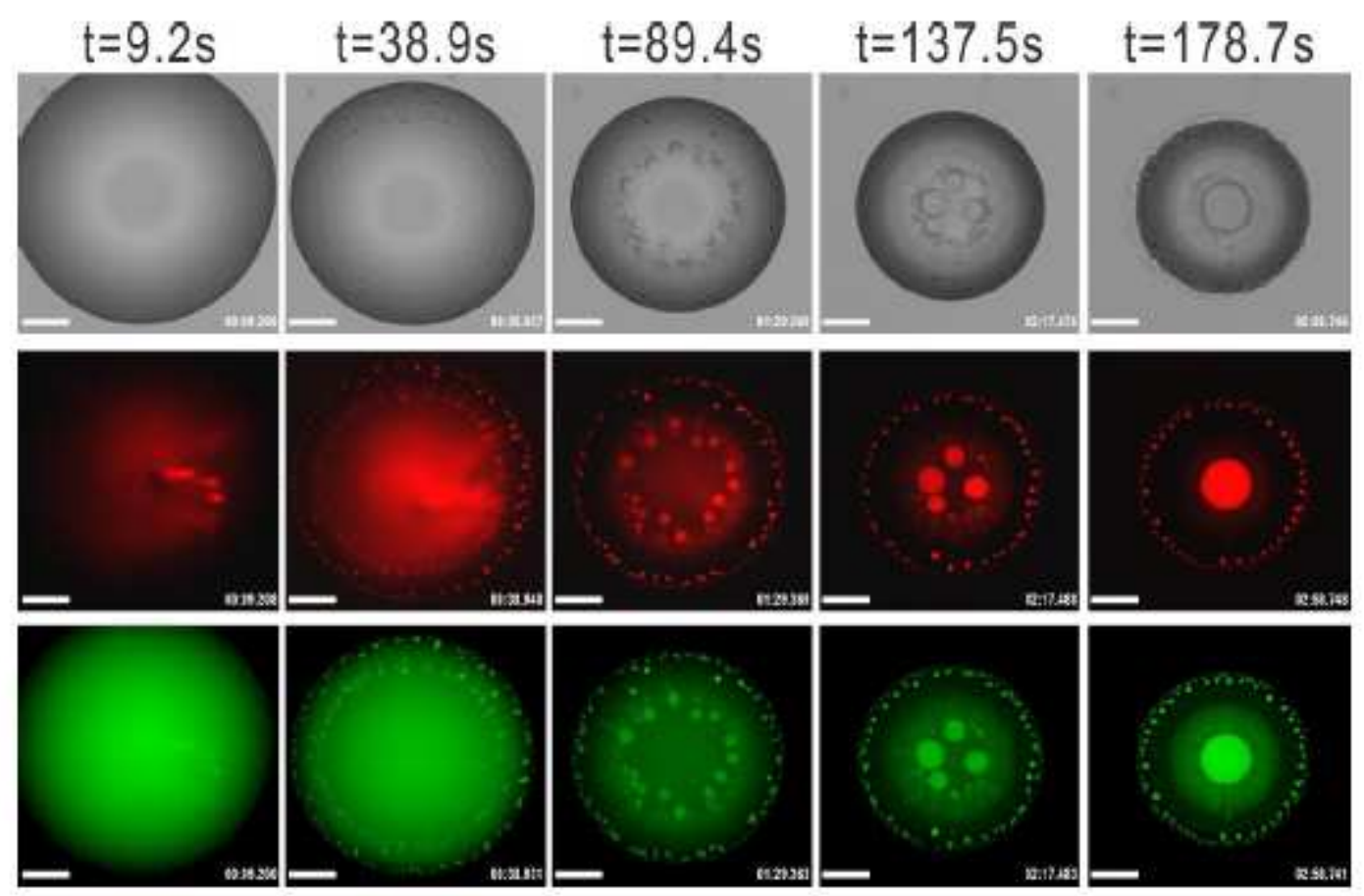

\section{Figure 3}

DNA localizes into dextran-rich compartments inside the sessile droplet. DNA is labelled with Cy-5 (red) and dextran-rich compartments are labelled with FITC-dextran (green). The scale bar is $500 \mu \mathrm{m}$.

(a)

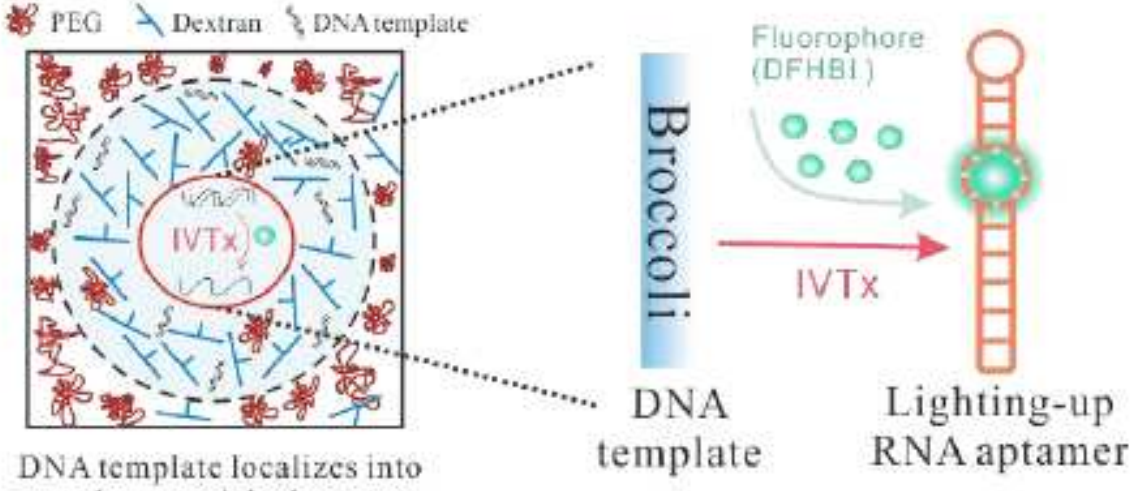
dextran-rich phase

(b1)

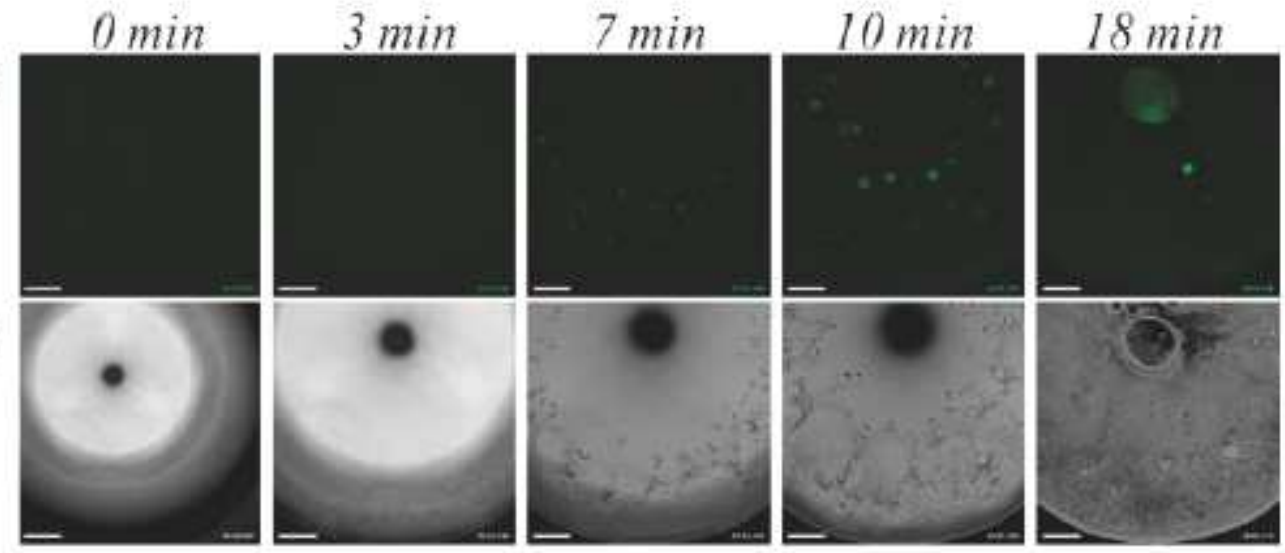

Figure 4 
(a) The flow of genetic information in phase-separated compartments via in vitro transcription (IVT). (b) Optical image sequence shows fluorescence RNA aptamer transcription from Broccoli DNA template inside the phase-separated compartments. The DNA templates are coded for Broccoli aptamer that can bind 5-difluoro-4-hydroxybenzylidene imidazolinone (DFHBI) to form a fluorescent complex of BroccoliDFHBI. The scale bar is $500 \mathrm{Dm}$.

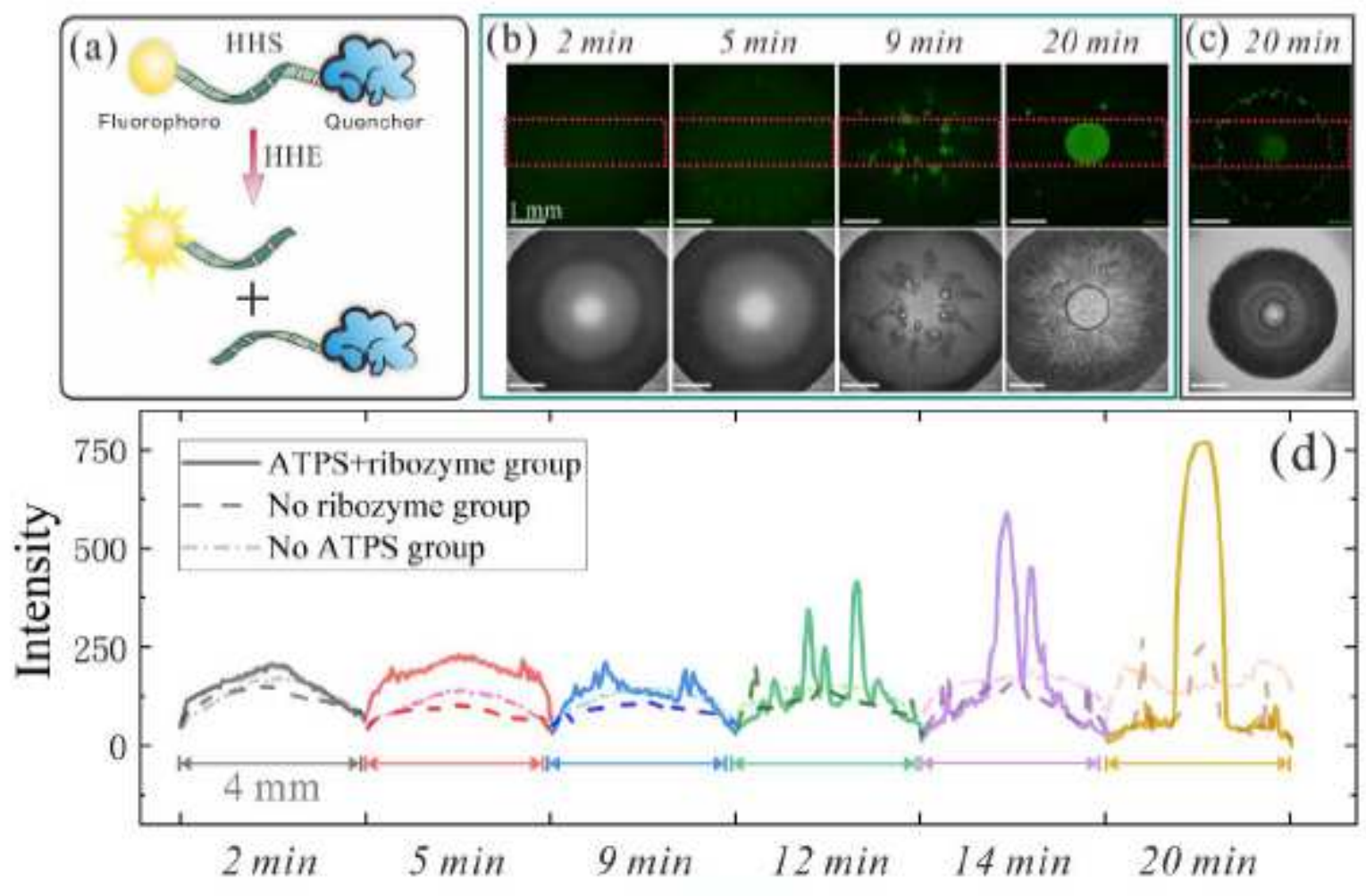

Figure 5

(a) Schematic drawing of the RNA cleavage reaction. The fluorophore labelled hammerhead substrate (HHS) is cut into two smaller pieces by the hammerhead ribozyme (HHE) and emits fluorescence. (b) Image sequence shows RNA cleavage process inside the phase-separated compartments. (c) Control group of the RNA cleavage reaction without HHE. There is only fluorophore labelled substrate added into the droplets. (d) Fluorescence intensity evolution of experimental group (solid line), no ribozyme control group (dashed line) as well as no ATPS control group (dash-dotted line). The intensity value is obtained by gel analysis of a specified rectangular area (as shown in (b) and (c)) in the fluorescence images. The scale bar is $1 \mathrm{~mm}$.

\section{Supplementary Files}

This is a list of supplementary files associated with this preprint. Click to download.

- MovieS1.mp4

- MovieS2.mp4 
- MovieS3.mp4

- MovieS4.mp4

- MovieS5.mp4

- MovieS6.mp4

- Supplementaryinformation.pdf 